\title{
Causally Ordered Message Delivery in Mobile Systems*
}

\author{
Sridhar Alagar and S. Venkatesan \\ Department of Computer Science \\ University of Texas at Dallas, Richardson, TX 75083 \\ $\{$ sridhar,venky\}@utdallas.edu
}

\begin{abstract}
There is a growing trend in using mobile computing environment for several applications, and it is important that the mobile systems are provided adequate support both at the systems level and at the communication level. Causal ordering is a useful property, particularly in applications that involve human interactions. In this paper, we present three algorithms for causal ordering in mobile systems. The first algorithm handles the resource constraints of the mobile hosts. But the system is not easily scalable and is not graceful to host disconnections and connections. The second algorithm eliminates the above disadvantages at the cost of inhibiting some messages. The third algorithm is a trade-off between the first two algorithms.
\end{abstract}

\section{Introduction}

Many of the distributed algorithms designed for static hosts cannot be directly used for mobile systems due to the change in physical connectivity, resource constraints of mobile hosts and limited bandwidth of the wireless links [1]. This has spawned considerable amount of research in mobile computing: designing communication protocols [3, 9], file system operations [4], and providing fault tolerance [5]. In this paper, we consider the problem of providing a particular kind of communication support, namely, causally ordered message delivery to mobile hosts. Consider two messages $m$ and $m^{\prime}$ sent to the same destination such that sending of $m$ "happened before" sending of $m^{\prime}$. Causal ordering is obeyed if $m$ is received before $m^{\prime}$ is received.

Causal ordering is useful in several applications like management of replicated data, resource allo-

*This research was supported in part by NSF under Grant No. CCR-9110177, by the Texas Advanced Technology Program under Grant No. 9741-036, and by grants from Alcatel Network Systems. cation, monitoring a distributed system, USENET etc., $[7,10]$. Causal ordering is best suited for applications that involve human interactions from several locations [10]; such applications are typical in mobile systems. Some of the major applications of distributed mobile systems in which causal ordering is useful are teleconferencing, stock trading, collaborative applications, etc.

\subsection{Motivation}

While designing algorithms for mobile systems, the following factors should be taken into account.

F1. The amount of computation performed by a mobile host should be low.

F2. The communication overhead in the wireless medium should be minimal.

F3. Algorithms should be scalable with respect to the number of mobile hosts.

F4. Algorithms should be able to easily handle the effect of hosts disconnections and connections.

If mobile hosts are made to execute the traditional causal ordering algorithms (by storing the relevant data structures in the mobile hosts), none of the above factors (F1-F4) can be satisfied. We present three algorithms for causal ordering in mobile systems.

Our first algorithm stores the data structures of mobile hosts (MHs) relevant to causal ordering in the mobile support stations (MSSs), and the algorithm is executed by the MSSs on behalf of the MHs. However, the message overhead is proportional to the square of the number of mobile hosts. Thus, factor F3 is not satisfied. Also, the algorithm is graceful to hosts disconnections and connections.

Algorithm 2 eliminates the problems in Algorithm 1. The size of the message header is proportional to the square of the number of MSSs. Since the size 
of the header does not vary with the number of mobile hosts, the algorithm is scalable (with respect to the number of the mobile hosts) and host disconnections/connections do not pose any problem. But there may be some "inhibition" in delivering the messages to the mobile hosts. Our experimental results suggest that delay due to inhibition is less than the delay involved in transmitting and processing the long header (of each message) used in Algorithm 1. Also, the load placed on the MSSs is less compared to Algorithm 1.

Algorithm 3 is a hybrid algorithm and is a tradeoff between algorithm 1 and algorithm 2. Every MSS is partioned into $k$ logical MSSs to reduce the delay due to "inhibition" in delivering the messages to MHs. However, $k$ cannot be large as this will increase the size of the header and hence the message overhead. A summary of our results is shown in Table 1.

\section{Model and Definitions}

A distributed mobile system consists of a set of mobile hosts and static hosts. A mobile host ( $\mathrm{MH})$ is a host whose geographical location can change with time while retaining its connectivity to the network [3]. A static host is a host whose location does not change during the computation. A static host can also be a mobile support station (MSS). An MSS has the necessary infrastructures to support the mobile hosts. For simplicity, we assume that the system consists of only MSSs and MHs. A static host can be considered as an MH that does not move.

The geographical area within which an MSS supports MHs is called a cell. Communication between MHs and MSSs is through a wireless channel. An MH can communicate directly with an MSS only if the MH is located in the cell of the MSS. A mobile host may belong to at most one cell at any time. Mobile hosts communicate with other hosts through their $\mathrm{MSSs}^{\dagger}$. MSSs are connected among themselves using wired channels. The MSSs and the wired channels constitute the static network. We assume that a logical channel exists between every pair of MSSs. These logical channels need not be FIFO channels; whereas the wireless channels are FIFO. Both wired and wireless channels are reliable and take an arbitrary but finite amount of time to deliver messages.

A mobile host can migrate from one cell to another cell at any time. Every MSS periodically broadcasts a beacon [3]. Let $\mathrm{MH} h_{1}$ move from the cell of MSS $s_{1}$

\footnotetext{
tThe MSS of an MH is the MSS in whose cell the MH is located.
}

to the cell of $s_{2}$. $h_{1}$ discovers that it is in the cell of $s_{2}$ after receiving the beacon broadcast by $s_{2}$. MH $h_{1}$ informs MSS $s_{2}$ of $h_{1}$ 's $i d$ and the $i d$ of its previous MSS $s_{1}$. A handoff procedure is then executed between $s_{2}$ and $s_{1} . s_{2}$ informs $s_{1}$ about $h_{1}$ 's migration and gets the relevant information associated with $h_{1}$ from MSS $s_{1}$.

A mobile host can disconnect itself from the network by sending a disconnect message to its current MSS and can reconnect at a later time by sending a connect message. If an MSSs receives a message for any of the disconnected mobile hosts, the message can be stored and delivered to mobile host after it reconnects, or the message can be dropped depending on the application.

An event in a host may be a send event (sending a message to another host), a receive event (receiving a message from a host), or an internal event which does not involve sending or receiving a message. Let send $(m)$ be the event that corresponds to the sending of message $m$ and $\operatorname{recv}(m)$ be the event that corresponds to the receipt of $m$. Events in a mobile system are ordered based on the "happened before" relation, $\rightarrow$, introduced by Lamport [6]. For any two events $e$ and $e^{\prime}, e \rightarrow e^{\prime}$ is true if (i) $e$ and $e^{\prime}$ are two events in the same host and $e$ occurs before $e^{\prime}$ or (ii) $e$ corresponds to sending a message $m$ and $e^{\prime}$ corresponds to the receipt of $m$ or (iii) there exists an event $e^{\prime \prime}$ such that $e \rightarrow e^{\prime \prime}$ and $e^{\prime \prime} \rightarrow e^{\prime}$. Causal ordering of message delivery is obeyed if, for any two messages $m$ and $m^{\prime}$ that have the same destination, $\operatorname{send}(m) \rightarrow \operatorname{send}\left(m^{\prime}\right)$ implies that $\operatorname{recv}(m) \rightarrow \operatorname{recv}\left(m^{\prime}\right)$.

\section{Preliminaries}

Causal ordering was first proposed for the ISIS system [2]. There are several algorithms that implement causal ordering for distributed systems with static hosts $[2,7,8]$. The algorithm by Birman and Joseph [2] appends, to every message, the history of the communications that happened before the sending of the message. The size of the appended information can become unbounded. However, the channels need not be reliable. The algorithm by Raynal, Schiper and Toueg, referred henceforth as RST algorithm, is based on message counting and assumes the channels to be reliable [7]. The RST algorithm, which we will discuss subsequently, appends $N^{2}$ integers to every message, where $N$ is the number of hosts in the system. The algorithm by Schiper et al. [8] uses vector clocks and is somewhat similar to the RST algorithm. In this paper we extend the RST algorithm to mobile systems. 


\begin{tabular}{|c|c|c|c|}
\hline Algorithm & Size of message header & "handof" Complexity & "inhibition" \\
\hline Algorithm 1 & $O\left(n_{h}^{2}\right)$ integers & $O(1)$ messages & No \\
Algorithm 2 & $O\left(n_{s}^{2}\right)$ integers & $O\left(n_{s}\right)$ messages & Yes \\
Algorithm 3 & $O\left(k^{2} * n_{s}^{2}\right)$ integers & $O\left(k * n_{s}\right)$ messages & decreases with $k$ \\
\hline
\end{tabular}

Table 1: $n_{h}$ is the number of MHs, $n_{s}$ is the number of MSSs, and $k$ is the number of logical MSSs per MSS. $n_{h}$ $\gg n_{s}$.

The RST algorithm for causal ordering maintains two arrays, $\operatorname{DELIV}_{i}[N]$ and $\operatorname{SENT}_{i}[N, N]$, for each host $P_{i}$. DELIV $_{i}[j]$ denotes the total number of messages received by $P_{i}$ from $P_{j}$. $\operatorname{SENT}_{i}[k, j]$ indicates $P_{i}$ 's knowledge about the number of messages $P_{k}$ has sent to $P_{j}$. The following steps are executed at $P_{i}$ to ensure causal ordering.

When $P_{i}$ sends message $m$ to $P_{j}, P_{i}$ appends its current value of $\mathrm{SENT}_{i}$ with $m$. $\left(P_{i}\right.$ sends $\left(m, \mathrm{SENT}_{i}\right)$ to $P_{j}$.) $P_{i}$ then increments $\operatorname{SENT}_{i}[i, j]$ by 1 .

On receiving $(m, S T)$ from $P_{j}$, the causal ordering algorithm at $P_{i}$ first checks if $\operatorname{DELIV}_{i}[k] \geq S T[k, i]$ for all $k$. If so, the message $m$ is delivered to the application, $\operatorname{DELIV}_{i}[j]$ is incremented by $1, \operatorname{SENT}_{i}[j, i]$ is set to $S T[j, i]+1$, and finally $\operatorname{SEnT}_{i}[j, k]$ is set to $\max \left(S T[j, k], \mathrm{SENT}_{i}[j, k]\right)$ for all $j, k$. If not, $m$ is queued till $\operatorname{DELIV}_{i}[k] \geq S T[k, i]$ for all $k$.

\section{Algorithm 1}

Algorithm 1 consists of two modules: static module and handoff module. The static module is executed when an MH is in a particular cell. The handoff module is executed when an MH moves from one cell to another.

\subsection{Static Module}

For each MH $h_{i}$, we maintain two arraysMH_DELIV $_{i}\left[n_{h}\right]$ and MH_SENT $i\left[n_{h}, n_{h}\right]$, where $n_{h}$ is the number of mobile hosts. MH_DELIV $i[j]$ denotes the total number of messages received by $h_{i}$ from $h_{j}$. MH_SENT $i[k, j]$ indicates $h_{i}$ 's knowledge about the number of messages $h_{k}$ has sent to $h_{j}$. Assume that MH $h_{i}$ is in the cell of MSS $s_{k}$. To reduce the communication and computation overhead of $\mathrm{MH} h_{i}$, these arrays are stored in MSS $s_{k}$. Since the messages from (to) $h_{i}$ go through MSS $s_{k}$, the causal ordering algorithm is executed by MSS $s_{k}$.

Initially, all the entries in the arrays MH_DELIV $i$ and MH_SENT $_{i}$ are set to 0 . To send a message $m$ to another MH $h_{j}, h_{i}$ first sends the message $m$ to its MSS $s_{k} . \quad s_{k}$ sends $\left(m, \mathrm{MH}_{-} \mathrm{SENT}_{i}\right)$ to the MSS of $h_{j}$ and increments MH_SENT $i[i, j]$. There are several protocols $[3,9]$ that ensure reliable message delivery to mobile hosts. Any of these protocols can be used.

MSS $s_{k}$, on receiving a message $(m, S T)$ meant for $h_{i}$ from MH $h_{j}$, first checks whether $m$ is deliverable. $m$ is deliverable if MH_DELIV $_{i}[k] \geq S T[k, i]$ for all $k$. If so, $s_{k}$ transmits $m$ to $h_{i}$, increments MH_DELIV $i[j]$, and MH_SENT $i[j, k]$ is set to $\max \left(S T[j, k],{\mathrm{MH} \_\operatorname{SENT}_{i}}_{i}[j, k]\right)$ for all $j, k . \quad m$ is also temporarily stored by $s_{k}$ in PEND_ACK ${ }_{i}$. Message $m$ will be deleted from PEND_ACK $i$ after receiving an ack for $m$ from $\mathrm{MH} h_{i}$. If $m$ is not deliverable, $m$ is stored in MH_PENDING $i$ till $m$ becomes deliverable. Whenever a message is delivered to $h_{i}, s_{k}$ checks MH_PENDING $_{i}$ for any message that becomes deliverable.

\subsection{Handoff Module}

Let $h_{i}$ move from the cell of MSS $s_{k}$ to the cell of MSS $s_{t}$. The handoff module is then executed by $s_{k}$ and $s_{t}$. After entering the cell of $s_{t}$, MH $h_{i}$ sends the message register $\left(h_{i}, s_{k}\right)$ to $s_{t}$. Also, $h_{i}$ retransmits the messages (to $s_{t}$ ) for which it did not receive ack from its previous MSS $s_{k}$. MSS $s_{t}$ then informs $s_{k}$ that $h_{i}$ has switched from MSS $s_{k}$ to MSS $s_{t}$ by sending a handoff_begin $\left(h_{i}\right)$ message to $s_{k}$. After receiving handoff_begin $\left(h_{i}\right), s_{k}$ transfers MH_DELIV ${ }_{i}$, MH_SENT $_{i}$, MH_PENDING $i$, and PEND_ACK $i$ to MSS $s_{t}$ and finally sends message handoff_over $\left(h_{i}\right)$ to $s_{t}$.

On receiving these data structures, $s_{t}$ first transmits all messages in PEND_ACK $i$. Also, $s_{t}$ forwards the messages (to their destinations) retransmitted by $h_{i}$. The handoff procedure is then terminated at $s_{t}$. If MH $h_{i}$ switches to some other cell before the handoff is completed, the current handoff is completed before a new handoff begins.

\subsection{Analysis}

For every message sent by MH $h_{i}$, the MSS (in whose cell $h_{i}$ resides) sends MH_SENT $i$ with the mes- 
sage. Hence, the size of the header for every message sent over the static network is $O\left(n_{h}^{2}\right)$ integers. The handoff module uses $O(1)$ messages of size $O\left(n_{h}^{2}\right)$ numbers when MH $h_{i}$ switches its cell.

Now, consider the factors F1-F4 discussed in Section 1.1. Since Algorithm 1 is executed at MSSs, factors $\mathrm{F} 1$ and $\mathrm{F} 2$ are satisfied. The overhead in the wireless medium is kept minimal. But factors F3-F4 are not satisfied. An overhead of $O\left(n_{h}^{2}\right)$ integers over the static network is costly if $n_{h}$ is very large. Also, due to disconnections and connections, $n_{h}$ varies. So during disconnections, some of the entries in the arrays MH_DELIV, and MH_SENT are not needed. The arrays need not be static, but maintaining dynamic arrays can become complicated if the $\mathrm{MH}$ disconnections and connections are frequent. In addition, the processing time for updating the matrix MH_SENT will be substantial for large $n_{h}$, and the nontrivial processing time increases the delay in delivering a message.

\section{Algorithm 2}

In Algorithm 1, messages are tagged with complete information to explicitly maintain causal ordering among the mobile hosts. In Algorithm 2, messages are tagged with sufficient information just to maintain causal ordering among the MSSs. Since the wireless channel between an MSS and an $\mathrm{MH}$ in its cell is FIFO, maintaining causal ordering at the static network level is sufficient if the MHs do not move. To ensure that causal ordering is not violated after an MH moves, we incorporate some steps into the handoff procedure.

\subsection{Static Module}

The static module is similar to the static module of Algorithm 1 but for some of the data structures. For each MSS $s_{i}$, we maintain MSS_DELIV $i\left[n_{s}\right]$, $\operatorname{MSS}_{-}$SENT $_{i}\left[n_{s}, n_{s}\right]$, and MSS_PENDING $i$. (This is unlike in Algorithm 1 where we maintain these data structures for every mobile host.) Observe that the size of the arrays MSS_DELIV $i\left[n_{s}\right]$ and $\operatorname{MSS} S E N T_{i}\left[n_{s}, n_{s}\right]$ vary with $n_{s}$, the number of MSSs. The value of MSS_DELIV $i[j]$ indicates the number of messages (whose destination can be different MHs) received from MSS $s_{j}$ by MSS $s_{i}$. MSS_SENT $i[k, j]$ denotes the number of messages sent by MSS $s_{k}$ (not necessarily delivered) to MSS $s_{j}$ that $s_{i}$ knows of. Every MSS knows (need not be exact) about the location of the MHs. Initially, we assume that the initial locations of MHs are known to all MSSs. We show how this knowledge gets updated in the next section. In other aspects, the static module is similar to the static module of algorithm 1 .

\subsection{Handoff Module}

The handoff module is more involved when compared to the handoff module of algorithm 1. Since causal ordering is explicitly maintained only at the MSSs level, some measures have to be taken during handoff to maintain causal ordering after an $\mathrm{MH}$ moves.

Before we describe the handoff module, we illustrate the problem at hand with an example. Consider mobile hosts $h_{1}, h_{2}$, and $h_{3}$. Assume that $h_{1}, h_{2}$ and $h_{3}$ are in the cells of MSSs $s_{1}, s_{2}$ and $s_{3}$ respectively. Let $h_{3}$ send a message $m_{1}$ to $h_{1}\left(m_{1}\right.$ will be sent to MSS $\left.s_{1}\right)$ and then send a message $m_{2}$ to $h_{2}$. Before receiving $m_{1}$, let $h_{1}$ switch to the cell of $s_{2}$. Now, MH $h_{2}$, after receiving $m_{2}$ from $h_{3}$, sends a message $m_{3}$ for $h_{1}$ to $s_{2}$. If $s_{2}$ delivers $m_{3}$ to $h_{1}$, causal ordering will be violated because $h_{1}$ has not yet received $m_{1}$. Also, $s_{2}$ cannot find out from the knowledge it has gained so far whether there are any in-transit messages for $h_{1}$ sent to $s_{1}$. However, if $s_{2}$ delivers $m_{3}$ after ascertaining that all the messages for $h_{1}$ sent to $s_{1}$ have been delivered, causal ordering will not be violated. Now, we describe the handoff module.

Assume that a mobile host $h_{k}$ switches from the cell of MSS $s_{i}$ to the cell of MSS $s_{j}$. After switching, $\mathrm{MH} h_{k}$ sends register $\left(h_{k}, s_{i}\right)$ message to $s_{j}$. On receiving this message, $s_{j}$ sends the message handoff_begin $\left(h_{k}\right)$ to $s_{j}$, and then broadcasts the message notify $\left(h_{k}, s_{i}, s_{j}\right)$ to all the MSSs. The message notify $\left(h_{k}, s_{i}, s_{j}\right)$ signifies that $\mathrm{MH} h_{k}$ has switched from MSS $s_{i}$ to MSS $s_{j}$. An MSS $s$, on receiving notify $\left(h_{k}, s_{i}, s_{j}\right)$ message, updates its local knowledge about the location of MH $h_{k}$ and sends a last $\left(h_{k}\right)$ message to $s_{i}$. After receiving notify $\left(h_{k}, s_{i}, s_{j}\right)$, MSS $s$ will send messages meant for MH $h_{k}$ only to $s_{j}$ (the new MSS of $h_{k}$ ) and not to $s_{i}$ (the previous MSS of $h_{k}$ ). MSS $s_{i}$, after receiving the message handoff_begin $\left(h_{k}\right)$ from $s_{j}$, sends enable $\left(h_{k}\right.$, PEND_ACK $k$ ) message to $s_{j}$ and waits for last $\left(h_{k}\right)$ messages from all the MSSs. Meanwhile, if any message received by $s_{i}$ for $h_{k}$ becomes deliverable to $h_{k}, s_{i}$ marks it as old and forwards it to $s_{j}$.

On receiving the message enable $\left(h_{k}, \mathrm{PEND} \_\mathrm{ACK} \mathrm{K}_{k}\right)$ MSS $s_{j}$ starts sending the application messages sent by $h_{k}$. Also, $s_{j}$ delivers all the messages in PEND_ACK $k$ in the FIFO order to $\mathrm{MH} h_{k} . s_{j}$ also delivers all the messages for $\mathrm{MH} h_{k}$ that are marked old to $h_{k}$ in the order in which the messages arrived. Any mes- 
sages for $h_{k}$ that are not marked old will be queued in MSS_PENDING $_{j}$.

MSS $s_{i}$ (the previous MSS of $h_{k}$ ), after receiving last $\left(h_{k}\right)$ from all the MSSs sends the message handoff-over $\left(h_{k}\right)$ to MSS $s_{j}$. Observe that no messages for $h_{k}$ sent to $s_{i}$ will be in transition after $s_{i}$ receives $\operatorname{last}\left(h_{k}\right)$ from all the MSSs. (Messages sent as part of handoff module are also causally ordered.) The handoff terminates at $s_{j}$ after handoff_over $\left(h_{k}\right)$ is received by $s_{j}$. If $s_{j}$ receives the message handoff_begin $\left(h_{k}\right)$ from some other MSS before the current handoff terminates (this can happen if $h_{k}$ switches its cell), $s_{j}$ will respond to the message only after the handoff terminates.

\subsection{Analysis}

The size of MSS_SENT is $n_{s}^{2}$ integers and hence the size of each message header over the wired network is $O\left(n_{s}^{2}\right)$ integers. The overhead does not depend on $n_{h}$, the number of MHs. Clearly, factors F3-F4 are satisfied. MH connections/disconnections do not affect the size of the arrays MSS_DELIV and MSS_SENT. During handoff, a notify message has to be sent to all the MSSs, and all the MSSs send last messages. Hence, the handoff module uses $O\left(n_{s}\right)$ messages. The storage requirement of Algorithm 2 and the load placed on the MSSs are less than that of Algorithm 1.

Though the handoff module is involved, it does not affect the performance (compared to Algorithm 1) due to the following reasons. (i) $\mathrm{MH} h_{k}$ does not wait for the handoff module to terminate to receive messages. It keeps receiving old messages. (ii) Messages sent by $h_{k}$ for other MHs are sent by $s_{j}$ (the new MSS of $h_{k}$ ) immediately after $s_{j}$ receives enable message.

The drawback of Algorithm 2 is the possibility of a message being "inhibited" from being delivered to an MH. There is an inhibition in delivering a message to an $\mathrm{MH}$ if it is queued in MSS_PENDING even though the delivery of the message does not violate causal ordering. Messages may be inhibited because, in Algorithm 2, causal ordering is explicitly implemented among the MSSs. Reception of a message may violate causal ordering from an MSS's point of view; whereas its delivery to an MH may not violate causal ordering from the MH's point of view. However, this delay is less than the delay introduced by Algorithm 1 in transmitting and processing the header of each message. The average delay in delivering a message in $\mathrm{Al}$ gorithm 2 is considerably less than the delay in Algorithm 1 when $n_{h}$ increases, as shown in Figure 1. (For the details of our simulation model, see Appendix A.) When $n_{h}<30$ the message header in both the algo-

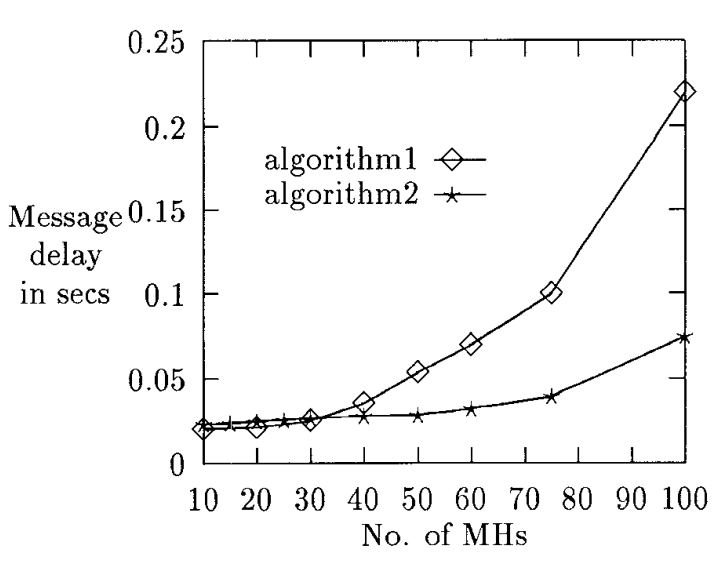

Figure 1: Comparison of Algorithm 1 and Algorithm 2 with respect to message delay. $n_{s}=10$

rithms are comparable in size. The message delay in Algorithm 2 is more than that of Algorithm 1 due to the inhibition inherent in Algorithm 2. However, as $n_{h}$ increases the delay due to processing the message header in Algorithm 1 dominates.

\section{$6 \quad$ Algorithm 3}

This algorithm reduces the delay in delivering the messages to $\mathrm{MH}$ due to inhibition, the drawback of Algorithm 2, without much increase in the message overhead. The algorithm achieves this by partioning every physical MSS into $k$ logical MSSs.

If an MH enters the cell of an MSS, the MH will be allocated to one of the logical MSSs depending on the load in each logical MSS of the MSS. The MHs will communicate with the other MHs through their logical MSSs. Every logical MSS maintains two arrays MSS_DELIV $\left[k * n_{s}\right]$ and MSS_SENT $\left[k * n_{s}, k * n_{s}\right]$ and a queue MSS_PENDING. The algorithm is the same as Algorithm 2 except for the fact that causal ordering is explicitly maintained among the logical MSSs. The size of the message header is $O\left(k^{2} * n_{s}^{2}\right)$.

Messages to MHs that belong different logical MSSs will not inhibit each other though the MHs may be in the same cell. Thus, as $k$ increases, the unnecessary delay in delivering the message to $\mathrm{MH}$ decreases. However, as $k$ increases the size of the message header will increase and, as a result, the time to process the message header will become a dominating factor. In Figure 2, the average message delay initially decreases 
when $k$ increases. But when $k$ becomes large the average message delay increases.

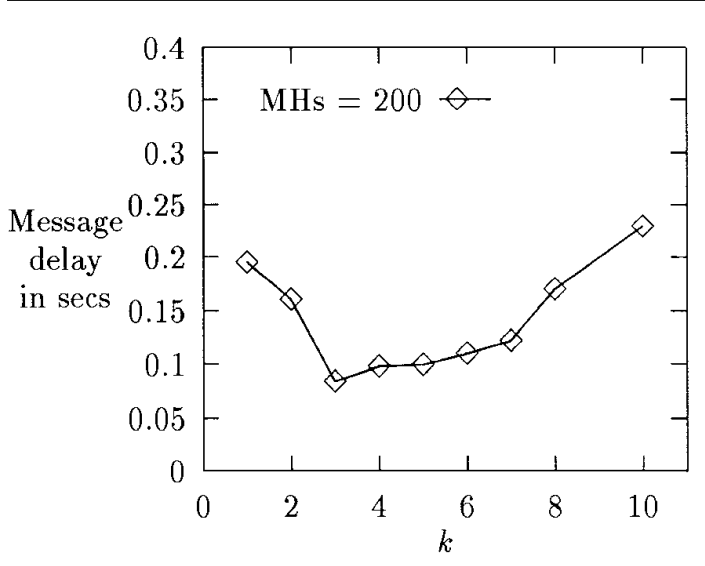

Figure 2: Message delays for various values of $k . n_{s}=$ 10.

\section{References}

[1] Badrinath, B., Acharya, A., And ImielinSKI, T. Impact of mobility on distributed computations. ACM Operating Systems Review 27, 2 (April 1993).

[2] Birman, K., and Joseph, T. Reliable communications in presence of failures. ACM Trans. Comput. Syst. 5, 1 (1987), 47-76.

[3] IoAnnidis, J., Duchamp, D., And Maguire, G. IP-based protocols for mobile internetworking. In Proceedings of ACM SIGCOMM Symposium on Communication Architecture and Protocols (1991), pp. 235-245.

[4] Kistler, J., and Satyanarayana, M. Disconnected operation in coda file system. $A C M$ Trans. Comput. Syst. 10, 1 (February 1992).

[5] Krishna, P., Vaidya, N., And Pradhan, D. Recovery in distributed mobile environments. In Proceedings of the IEEE Workshop on Advances in Parallel and Distributed Systems (1993), pp. 83-88.

[6] Lamport, L. Time, clocks, and the ordering of events in a distributed system. Commun. $A C M$ 21,7 (1978), 558-565.
[7] Raynal, M., Schiper, A., And Toueg, S. Causal ordering abstraction and a simple way to implement it. Inf. Process. Lett. 39, 6 (1991), 343-350.

[8] Schiper, A., Eggli, J., And Sandoz, A. A new algorithm to implement causal ordering. In Proceedings of the 3rd International Workshop on Distributed Algorithms (1989), Springer Verlag, pp. 219-232.

[9] Teraoka, F., Yokote, Y., and Tokoro, M. A network architecture providing host migration transparency. In Proceedings of ACM SIGCOMM (September 1991).

[10] VAn Renesse, R. Causal controversy at le mont st.-michel. ACM Operating Systems Review 27,2 (April 1993), 44-53.

\section{A Simulation Details}

Our simulation model is similar to that of in [5]. The simulation is event driven and it is run on a Sparc 10 station. The events are send message, receive message, and handoff. The bandwidth of a wired channel is assumed to be $100 \mathrm{Mbits} / \mathrm{sec}$, and the propagation delay in a wired channel is $7 \mathrm{~ms}$. For a wireless channel, the bandwidth and propagation delay are assumed to be $1 \mathrm{Mbits} / \mathrm{sec}$ and $500 \mu \mathrm{s}$, respectively.

Initially, the cells of the mobile hosts are assigned randomly. The time interval between two send events in a mobile host is an exponentially distributed random variable with a mean of $t_{s}$ seconds. The time interval between handoff is also an exponentially distributed random variable with a mean of $t_{h}$ seconds. The values of $t_{s}$ and $t_{h}$ are varied $(0.1,1.0,10 \mathrm{secs})$ to consider different scenario of communication and mobility. The processing time considered in measuring the message delay is the actual CPU running time in processing the message header. The value of every point in the graph is an average of the results of 1000 experiments performed. 